\title{
Entropic Entanglement: Information Prison Break
}

\author{
Alexander Y. Yosifov and Lachezar G. Filipov \\ Space Research and Technology Institute, Bulgarian Academy of Sciences, Sofia, Bulgaria \\ Correspondence should be addressed to Alexander Y. Yosifov; alexanderyyosifov@gmail.com
}

Received 13 January 2017; Accepted 19 April 2017; Published 4 July 2017

Academic Editor: George Siopsis

Copyright (c) 2017 Alexander Y. Yosifov and Lachezar G. Filipov. This is an open access article distributed under the Creative Commons Attribution License, which permits unrestricted use, distribution, and reproduction in any medium, provided the original work is properly cited. The publication of this article was funded by SCOAP ${ }^{3}$.

\begin{abstract}
We argue that certain nonviolent local quantum field theory (LQFT) modification considered at the global horizon $(r=2 M)$ of a static spherically symmetric black hole can lead to adiabatic leakage of quantum information in the form of Hawking particles. The source of the modification is (i) smooth at $r=2 M$ and (ii) rapidly vanishing at $r \gg 2 M$. Furthermore, we restore the unitary evolution by introducing extra quanta which departs slightly from the generic Hawking emission without changing the experience of an infalling observer (no drama). Also, we suggest that a possible interpretation of the Bekenstein-Hawking bound as entanglement entropy may yield a nonsingular dynamical horizon behavior described by black hole thermodynamics. Hence, by treating gravity as a field theory and considering its coupling to the matter fields in the Minkowski vacuum, we derive the conjectured fluctuations of the background geometry of a black hole.
\end{abstract}

\section{Introduction}

It has been argued in [1] that black holes are not black at all. Rather, in a semiclassical approximation they are shown to be hot bodies that emit thermal radiation with an inverse temperature of $2 \pi$. The fate of information fallen into a black hole is still under debate. Hawking's original proposal of loss of information and thus pure-to-mixed state evolution has been strongly opposed [2-11] as it implies violation of quantum-mechanical unitarity. Resolution of the information paradox within the current nomenclature does not seem to be a fruitful endeavor. Instead, we have focused on modifying already existing principles. Abandoning locality above the Planck mass $\left(m_{p}\right)$, for instance, appears as a promising and somewhat more conservative approach. Significant theoretical support for fundamental nonlocality has come from AdS/CFT duality and cosmology [12-16]. One of the authors [16] has shown that in the extreme conditions of the early universe (super-Planckian energies) nonlocality plays an essential role for explaining the origin of the cosmological principle.

Following the theoretical evidence, in the current paper, we embrace the notion of locality as an effective field theory, manifesting in weak gravitational dynamics. Based on that assumption we propose a framework, featuring a modification of local quantum field theory as defined on the global horizon $(r=2 M)$ in order to provide a nonviolent mechanism for taking the Hawking quanta (quantum information) out of the hole, and thus restore unitarity. The suggested LQFT modification leads to weak (nonviolent) quantum effects which manifest in brief nonlocal phenomena. Despite being weak, the perturbative nature of the effects makes them significant in the course of a black hole's lifetime $O\left(M^{3}\right)$. The current model also predicts minor deviations from the generic Hawking emission sufficient to restore the unitary evolution without causing drama for an observer in free fall.

By treating gravity in a black hole background metric (Minkowski space) as a field theory (graviton) we derive the microscopic origin of the conjectured Planckian-amplitude horizon oscillations [17]. In a previous paper we approached the phenomena classically by deriving the oscillations from perturbation theory (see [17]). The results we obtain in the particular work may be considered as a microscopic origin of the stretched horizon in observer complementarity [18]. Furthermore, the effects of the source of the LQFT modification are shown to respect the equivalence principle and also to rapidly vanish at large $r$. 
The paper is organized as follows. In Section 2 we put forward the LQFT revisions and show how the coupling between the graviton and the matter fields in Minkowski space can lead to information escape. In Section 3 we provide a quantum theory derivation of the proposed horizon oscillations by treating gravity in the vicinity of a black hole as field theory.

\section{Nonlocal Information Release}

The particular modification of LQFT, which we propose, comes from localized and brief violations of locality, yielded by "strong" fluctuations of the graviton that come from its coupling to the matter fields in Minkowski space. We also put forward a gedanken experiment which involves a pair of strings put on both sides of the future Rindler horizon in order to show that the equivalence principle remains valid for an observer in free fall.

Based on the thermal spectrum of the emitted radiation and black hole thermodynamics (Second Law, in particular) we now take for granted the proportionality between black hole entropy and horizon area. Namely, the entropy of a black hole is one-fourth of the area of the event horizon in Planck units:

$$
S_{\mathrm{BH}}=\left(\frac{A}{4}\right)
$$

The geometric entropy bound is deeply rooted in holography and further generalized in the Bousso bound [19]. Yet, its origin has not been fully explained.

2.1. Geometric Entropy = Entanglement Entropy. We begin by showing how the Bekenstein formula can be derived from entanglement entropy and later how this can yield the small departures from LQFT needed to carry the quantum information out of the black hole.

Bianchi has shown [20] that by considering the correlations between gravity and matter fields in the near-horizon region one can reproduce the Bekenstein-Hawking bound equation (1). The obtained equality has been shown to be universal and independent of the number of field species. Hence Bianchi's derivation of the equality $S_{\text {ent }}=S_{\mathrm{BH}}$, where $S_{\text {ent }}$ is entanglement entropy, shows that quantum entanglement is the fundamental origin of the Bekenstein entropy bound. Let us further clarify that.

Imagine we have a Schwarzschild black hole in a pure state $|\Psi\rangle$ with metric

$$
\begin{aligned}
d s^{2}= & -\left(1-\frac{2 M}{r}\right) d t^{2}+\left(1-\frac{2 M}{r}\right)^{-1} d r^{2} \\
& +r^{2}\left(d \theta^{2}+\sin ^{2} \theta d \varphi^{2}\right),
\end{aligned}
$$

where the singularity is at $r=0$ and the global horizon is at $r=2 M$.

Here, a black hole event horizon provides a perfect entangling surface as it naturally causally disconnects the interior and exterior regions

$$
\mathscr{H}=\mathscr{H}_{A} \otimes \mathscr{H}_{B},
$$

where $\mathscr{H}_{A}$ is the interior region $(r<2 M)$ and $\mathscr{H}_{B}$ is the exterior $(r>2 M)$. The dimensionality of $\mathscr{H}_{A}$ is given as the logarithm of the internal degrees of freedom.

The pure state of the complete system is given by the product of the two subsystems

$$
|\Psi\rangle=\sum_{i}\left|A_{i}\right\rangle \otimes\left|B_{i}\right\rangle
$$

with a corresponding density matrix

$$
\rho=|\Psi\rangle\langle\Psi|
$$

The pure state of the complete system may be decomposed as

$$
|\Psi\rangle \longrightarrow \rho_{A}+\rho_{B}
$$

where $\rho_{A}$ and $\rho_{B}$ denote the reduced density matrices of the corresponding subsystems $A$ and $B$, respectively. Note that in a black hole background the initial state cannot be trivially reproduced by the thermal density matrices; some of the information concerning $|\Psi\rangle$ is found in the entanglement between the two subsystems across the entangling surface.

Consider the Minkowski vacuum in the region near the black hole which is bounded by a local Rindler horizon $\mathscr{H}^{+}$. The complementary left and right Rindler wedges, described by the Hilbert spaces $\mathscr{H}_{A}$ and $\mathscr{H}_{B}$, respectively, are given in terms of thermal density matrices; see (6). The complete vacuum state $\rho_{0}$ is due to the entangling between the field theories defined on both sides of the horizon ( $L$ and $R$ Rindler wedges):

$$
\rho_{0}=\sum_{i} e^{-\beta}\left|E_{i}^{A}\right\rangle \otimes\left|E_{i}^{B}\right\rangle
$$

where $\left|E_{i}{ }^{A}\right\rangle$ and $\left|E_{i}{ }^{B}\right\rangle$ are the eigenstates associated with the wedges and $\beta$ denotes the inverse temperature.

In the vacuum state every mode on the left Rindler wedge is entangled with the corresponding mode on the right wedge. The particular entanglement normalizes the stressenergy tensor at $r=2 \mathrm{M}$, and hence an infalling observer does not feel anything out of the ordinary. The stress tensor normalization provides a smooth transition between the two distinct causal patches. Thus the entanglement entropy is proportional to the entangling surface (horizon). As a result, only modes very close to the global horizon contribute to the entropy of the system.

Bianchi's derivation of the equality $S_{\mathrm{ent}}=S_{\mathrm{BH}}$ strongly advocates the entanglement origin of the geometric entropy. Considering the correlations between gravity and matter fields in Minkowski space suggests we can treat gravity as a field theory (graviton). In particular, we argue that by treating gravity in Minkowski space in terms of quantum field theory in black hole background we can obtain the desired modification of local quantum field theory in the vicinity of the horizon and thus present a framework for gradual release of quantum information.

2.2. Gravity as a Field Theory. Suppose we assign a timedependent Killing frequency to the graviton with respect 


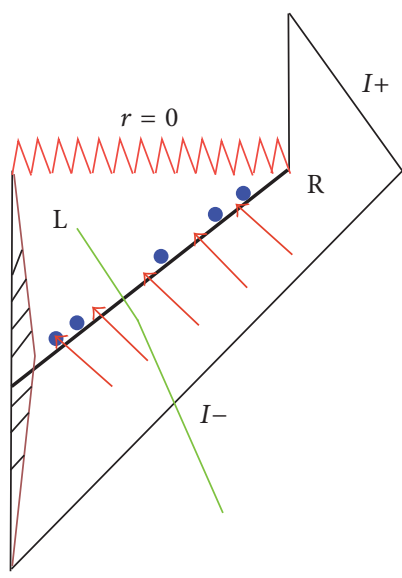

Figure 1: Penrose diagram depicting the matching between the randomly embedded qubits (blue dots) and strong fluctuations (red arrows). The wave-like red line is the singularity $(r=0)$, the solid black line is the global horizon $(r=2 M)$, and the green line is the worldline of an infalling observer. $L$ and $R$ stand for left and right Rindler wedge, respectively. $\mathscr{L}^{-}$and $\mathscr{L}^{+}$denote past and future null infinity, respectively.

to the background metric. The correlated quantum fields (gravity and matter) in the near-horizon region oscillate rapidly and have radial dependence with respect to the Rindler horizon $\mathscr{H}^{+}$. Generally, the quantum fluctuations of the matter fields near the horizon get "amplified" by the black hole's internal degrees of freedom and an inertial observer with a measuring apparatus in that region measures $\left\langle N_{i}\right\rangle=\langle 0|N| 0\rangle$, where the expectation value of $N$ is nonzero (Hawking process).

We wish to focus on the black hole metric back-reaction from the graviton fluctuations in two cases (i) Minkowski space and (ii) the vicinity of the horizon. Since we consider locality in effective field theory to be a constraint imposed by the background geometry, we wish to examine how fluctuations of the gravitational field above a threshold $\lambda$ affect it.

We believe that strong quantum fluctuations of the gravitational field as considered onto the horizon $(r=2 M)$ will cause "disturbances" in the background metric and the effective field theory description (locality). Consider the following gedanken experiment. Imagine we have a static black hole; see (2) and Figure 1.

For convenience when describing the strong graviton fluctuations we will consider a time slice on which the mass of the hole is time-translation invariant. Hence, the influx of matter exactly matches the emission of Hawking particles to future null infinity. In the particular case the emission should be thought of as a statistical phenomena which solely depends on the internal Hilbert space $\mathscr{H}_{A}$. However, small deviations may appear due to the random nature of the fluctuations (excitations). Also, consider the following assumptions: (i) the future Rindler horizon $\mathscr{H}^{+}$is given in terms of null light rays which neither get inside the black hole nor get emitted to asymptotic infinity, $r<2 M<\mathscr{H}^{+}<r>2 M$, (ii) black holes act as fast scramblers [21] and information is found in the emitted quanta, and (iii) scrambled information need not be embedded uniformly across the horizon as this would make $\log R<R \log R$, and, as a result, an outside observer would be able to verify a violation of the linearity of quantum mechanics. Note that in a recent paper [22] we reproduced (with different initial assumptions and approach) the results of Page [23] regarding information distribution onto the horizon.

That being said, suppose we place two corresponding strings on both sides of $\mathscr{H}^{+}$. Imagine we have one string [24] with randomly placed qubits on it located very close to the global horizon $r<2 M$. Particularly, suppose the randomly distributed scrambled qubits [21] in the left Rindler wedge (Figure 1) are interpreted as switches turned on. Similarly, the string on the corresponding right wedge $(r>2 M)$ is said to have statistical graviton excitations which can also be interpreted as switches, however, with one subtle distinction. We treat the fluctuations as switches turned on if their energy density exceeds a certain threshold $\lambda$. More precisely we have the following.

(A) We think of a fluctuation of the graviton as strong (switch on) if, when considered at asymptotic spatial infinity, its local energy density can polarize the vacuum and thus produce a particle $a_{i}^{\dagger}|0\rangle=|x\rangle$. Therefore, we can associate the strong fluctuations in a given space-time region with the expectation value for particle production $\left\langle N_{i}\right\rangle$ in that region. Generally

$$
\sum_{i=1}^{N} \int_{\Sigma} \varphi_{\text {strong }}=\left\langle N_{i}\right\rangle,
$$

where $\varphi_{\text {strong }}>\lambda$.

(B) The fluctuations below that threshold, thus $\varphi_{\text {weak }}<\lambda$, are considered weak (switch off). Namely, $a_{i}|0\rangle=|0\rangle$.

Reference [25] provides a good description of the pair of corresponding strings, acting on both sides of the Rindler horizon; namely, for an infalling observer we get

$$
\int_{\Sigma} O_{L} \varphi_{L R} O_{R}
$$

where the operators $O_{L}$ and $O_{R}$ are the corresponding ones for the left and right Rindler wedges, respectively, and $\varphi_{L R}$ is the source (graviton) which depends on the internal degrees of freedom of the black hole.

We are interested in the quantum effects which arise when there is a correspondence in the relative states of the occupation numbers on both sides of $\mathscr{H}^{+}$. That is a strong fluctuation in $R$ region and a qubit in $L$ region, where both are on the same Cauchy surface

$$
|00\rangle+|11\rangle \text {. }
$$

Let us consider a portion of the Rindler-like horizon $R_{H}$, where $R_{H} \ll A_{H}$ and $A_{H}$ is horizon area

$$
\int_{R_{H}} \delta \varphi_{\text {graviton }}{ }^{(x)},
$$

where the graviton is $\varphi$ and $\delta$ denotes the variations of the field. 
So a strong fluctuation $(\varphi>\lambda)$ corresponding to an embedded information (switch on) (Figure 1) would briefly disturb the local dynamics of the background metric and thus yield $[\varphi(x), \varphi(y)]=0$. As a result, the corresponding mode, placed on the inner string (left Rindler wedge), will be radiated to $\mathscr{I}^{+}$as a low $T$ Hawking particle. The "brief" disturbance should be of order of the lifetime of the fluctuation $\varphi$ and should not lead to significant changes in the background metric. Since the modifications are considered onto $r=2 M$, the near-horizon physics is consistent with the postulates of complementarity (Postulate II, in particular) [18], and thus the nonlocal effects are completely nonviolent for an observer close to the horizon. For instance, suppose that Alice is in the Minkowski vacuum carrying a measuring apparatus. When she performs measurements, she will see the typical effective field theory correlations between the exterior and interior of the black hole. Complementarily, Bob, being far away from the black hole will not see any significant deviations from the semiclassical Hawking framework.

We now examine the results from treating gravity as a field theory and considering the fluctuations which arise from its universal coupling to the matter fields in the vicinity of the horizon.

2.2.1. No Firewall. As it has been shown in [25] the desired LQFT modification which arise from the universal coupling between graviton and matter fields in the near-horizon region $\int \varphi^{\mu \nu} T_{\mu \nu}$ need not stop at $r=2 M$ but rather extend beyond the horizon in order to avoid firewall formation. Giddings argued (see [25]) the source of any such effects need to obey certain constraints:

(i) Smooth behavior at $r=2 \mathrm{M}$

(ii) Rapidly vanishing outside "the zone"

In what follows we show that the conjectured horizon fluctuations $[17,22]$ satisfy both conditions.

Let us begin with the latter constraint. The source (graviton) depends on the internal degrees of freedom which can be taken as invariant on a particular time slice. Thus it is trivial to show the radial- and time-dependence of the graviton and how it vanishes rapidly at large $r$ given the definitions we have provided regarding weak and strong fluctuations. Consider the following gedanken experiment. Imagine an isolated black hole and an observer coming from past null infinity who is carrying a measuring apparatus and constantly performing measurements. Suppose now she gets close to the black hole and then accelerates to future null infinity. We wish to know what the radial dependence of the measurements is. Since the graviton is treated in terms of the Hawking formalism, we assume the expectation value of the measurements to rapidly decrease at large $r$. Thus $\left\langle N_{i}\right\rangle=$ $r^{-1}$ with the transition being continuous. That is to say that outside "the zone" $(r \gg 2 M)$ the graviton fluctuations are $\ll \lambda$.

The former requirement is satisfied as follows. The nonlocal effects arising from the field theory treatment of the graviton are expected to cause no drama for an observer crossing the horizon since (i) they have a lifespan of order

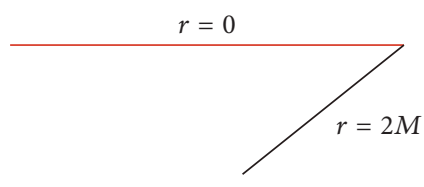

FIGURE 2: Diagram of the near-horizon region.

of the fluctuation $\varphi$ and (ii) are considered onto the horizon and are thus spacelike separated from an observer in Rindler space. Consider the following gedanken experiment. Suppose we examine the given graviton fluctuations by focusing on a neighborhood of the Rindler-like horizon (Figure 2).

In particular, let us think of the field fluctuations $\varphi$ in terms of harmonic oscillators (HOs). More precisely, imagine we place harmonic oscillators onto $\mathscr{H}^{+}$with relatively small spacing $\epsilon$ in-between

$$
\sum_{n=1}^{N} \int_{R} d \varphi\left(n_{i} \varphi_{i}\right)
$$

where $R$ denotes the horizon region, and $\varphi$ is the frequency. The net number of harmonic oscillators is given by $N$, where for a fixed cut-off $N \sim A_{H} / \epsilon$.

Note the individual HOs need not have the same radial frequency $\varphi$. The cut-off $\epsilon$ should be taken within an arbitrary distance spectrum; hence it must be normalized. Again, we are interested in harmonic oscillators with oscillation frequencies above a certain threshold $\lambda$. The influence ("domain of dependence") of those higher frequency HOs is highly localized and thus proportional to their sizes which are considered to be $\gg l_{p}$. As a result, an individual harmonic oscillator with arbitrary high frequency cannot affect significantly the horizon geometry.

Note the conjectured highly localized and brief violations of locality occur only in the presence of horizon since it foliates the given space-time region into distinct causal patches with continuous transition between the corresponding CFTs; see (7).

2.2.2. Extra Quanta. Restoring the unitary evolution of a black hole in generic models requires extra quanta to be emitted. Moreover, we wish the Hawking emission deviations to be consistent with the postulates of observer complementarity, hence no divergence of the stress tensor at the horizon. Again, we focus on the coupling between graviton and matter fields in the near-horizon region $\int \varphi^{\mu \nu} T_{\mu \nu}$ and argue that, by making certain conservative assumptions, an extra quantum per time $R$ is achievable in the current framework without the need of introducing additional degrees of freedom at the horizon (firewall). Namely, by assuming (i) a black hole begins to decay much before Page time ( $\left.\ll t_{\text {Page }}\right)$, (see [22]) and (ii) the extra quantum which carries out the quantum information is of longer wavelength $(\sim R)$, smooth horizon can be easily achieved even in the case of an extra particle per $R$. In this case the additional modes will only appear as a small correction in the overall perturbation. Note the needed quanta are very few; an extra particle per time $R$ is 
sufficient to restore the unitary evolution. In [22] we argued, by postulating assumptions regarding the internal dynamics of a static black hole, that quantum information emission in the form of Hawking modes can begin $O(R \log R)$ after the collapse, where $R \log R \ll t_{\text {Page }}$. The additional modes have low Hawking temperature of $\sim 1 / R$. Provided information begins leaking $\ll t_{\text {Page }}[22,23]$; then the entanglement entropy of the black hole will be negligible compared to the Bekenstein-Hawking bound. Generically, the extra modes wavelengths are comparable to the horizon radius $\approx R$ and are thus considered "soft." However, even if the energy density of the additional particles exceeds the Hawking temperature by a certain factor, there will be still no drama for an infalling observer. Moreover, the current framework agrees with the Page spectrum concerning the adiabatic information release from a black hole which has evaporated less than half of its coarse-grained entropy. Therefore, for a perturbed "young" black hole after $R \log R$ the emission rate is

$$
\frac{d I}{d t} \sim \exp \left[-\frac{4 \pi}{y^{2}}\right]
$$

where $y^{2}=m_{p} / E$ and $E$ is the energy of the emitted radiation.

\section{Horizon Oscillations from Weak Fluctuations}

In the current Section we focus on the weak fluctuations of the graviton very near the global horizon and specifically how they can lead to the conjectured Planckian-amplitude horizon oscillations [17]. In particular, we are interested in the back-reaction of the global horizon due to fluctuations below the threshold $\lambda$. Considering the coupling between gravity and matter fields in a black hole space-time leads to nontrivial dynamics of $\mathscr{H}^{+}$due to the "amplified" energy density of the fluctuations by the internal degrees of freedom of the hole. The effect of black hole's mass on the surrounding fields is best illustrated by the Hawking effect.

Let us begin by defining what we mean by weak fluctuations $(\varphi<\lambda)$. Similar to the definition of strong fluctuations $(\varphi>\lambda)$ provided in Section 2, we define a weak fluctuation of the graviton to be one that, when considered at asymptotic spatial infinity, does not lead to a particle production. Hence the expectation value of $N_{i}$ vanishes. Suppose Bob carries a very sensitive apparatus which can detect excitations with arbitrary low energy density and stays far away from the black hole. In general, we suspect Bob should measure negligibly small number for $N_{i}$ compared to $\varphi_{\text {weak }} ;$ namely, $\lambda \gg\left\langle N_{i}\right\rangle$. Since the weak fluctuations cannot affect significantly the background metric and the effective field theory description, they may be thought of as a source of smaller geometrical disturbances, hence horizon oscillations (fluctuations). We initially derived the horizon oscillations [17] from perturbation theory and argued that they followed the thermodynamic evolution of the hole. The frequency of the horizon oscillations is given as

$$
\omega=\left(\frac{-T_{\mu \nu}}{M_{\mathrm{BH}}}\right)^{3 / 2}
$$

where $T_{\mu \nu}$ is the stress tensor or the radiated Hawking particles.

The oscillations occur naturally in the process of black hole formation/evaporation and are expected in any physically meaningful theory of quantum gravity. During the evaporation of a black hole we assume

$$
\frac{d^{2} A(t)}{d t^{2}}=-T_{\mu \nu}
$$

Note that $T_{H}=1 / M_{\mathrm{BH}}$.

There are certain constraints that the dynamics which take care of information escape to asymptotic infinity need to respect. If we wish to keep LQFT in Minkowski space and simultaneously have spacelike transfer of quantum information a firewall will form. For that reason Giddings has argued [26] that if we wish to avoid formation of a firewall, $T_{\mu \nu} \rightarrow \infty$ as $r \rightarrow 2 M$, the modified LQFT must extend beyond the horizon. Thus, the conjectured horizon fluctuations may serve as the microscopic origin of the desired effects which normalize the experience of an infalling observer. We can illustrate the effects of the weak graviton fluctuations on the background geometry in terms of the strings introduced in Section 2. Consider now a string just outside the global horizon with harmonic oscillators placed on it; see (12). Since $\varphi_{i}$ are taken to be below $\lambda$ we expect them to yield metric fluctuations of order of the Planck length. For a comprehensive review of the conjectured Planckianamplitude metric fluctuations see [17].

Further, several authors [27-31] have suggested horizon oscillations (fluctuations) are generic phenomena in quantum gravity. Let us briefly comment on the existing literature. First, in [27], Bekenstein and Mukhanov proposed that horizon fluctuations may be achieved by describing the black hole as a quantum system of discrete energy levels. In the process of decay, the black hole "jumps" from one energy level to the next and thus smears the precise location of the horizon. Although the approach may seem different than the classical one we have proposed [17], or the currently considered, the results appear to be identical. Namely, given the particular entropy spacing between the different energy levels and the transition between them, they have derived a frequency equation almost identical to (14). As a consequence, beyond a certain threshold for $\omega$, no radiation will be further emitted [32]. Furthermore, for a thermal emission the mass dependence on $T_{\mu \nu}$ appears identical to (15). Mathur argued in [33] that oscillations of the horizon surface may be derived from "hard impacts" in the context of fuzzball complementarity. More precisely, high energy infalling quanta $E \gg T$, where $T$ is the local Hawking temperature, impact the fuzzball surface of the hole and cause oscillations which wear out and produce low-temperature Hawking particles $E \sim T$ which are supposed to carry out the quantum information. The framework should be contrasted to the particular paper since the horizon oscillations in our work result from the generic coupling between the graviton and the fields of nature in Minkowski space. Moreover, the model we put forward achieves unitary evaporation without diverging the stress tensor at the horizon. It was shown in [34] 
that subtle modifications of LQFT can indeed lead to emission of quantum information to asymptotic infinity without the formation of a firewall. For an explicit derivation of how gravitational collapse of massive shell in a semiclassical background geometry leads to pure state density matrix see [35].

\section{Conclusions}

By embracing the notion of locality as an effective theory and by treating gravity in Minkowski space as a field theory, we presented a scenario for adiabatic information release from a static black hole which does not cause drama for an infalling observer and begins much before Page time. Namely, by introducing extra particle radiation beyond the Hawking emission we manage to restore the unitary evolution without forming a firewall. Further, the current framework does not lead to divergence of the stress-energy tensor at the global horizon due to the early emission initiation and the low energy density of the emitted particles. The model presents perturbative quantum effects which emerge from considering strong fluctuations $(>\lambda)$ of the gravitational field at the global horizon, namely, highly localized and brief disturbances of locality, as imposed by the background metric. On the other hand, by focusing on the graviton fluctuations below the threshold $\lambda$ very near and at the horizon, we provided a microscopic origin of the conjectured Planckian-amplitude horizon oscillations (fluctuations). Therefore, entanglement appears to be the origin of the horizon fluctuations. Entanglement continues to show its importance, not only in the context of emergence of space and time, but also in quantum gravity phenomena in black holes.

\section{Conflicts of Interest}

The authors declare that there are no conflicts of interest regarding the publication of this paper.

\section{References}

[1] S. W. Hawking, "Breakdown of predictability in gravitational collapse," Physical Review D, vol. 14, no. 10, pp. 2460-2473, 1976.

[2] D. N. Page, "Is black-hole evaporation predictable?" Physical Review Letters, vol. 44, no. 5, pp. 301-304, 1980.

[3] G. 't Hooft, "On the quantum structure of a black hole," Nuclear Physics B, vol. 256, pp. 727-745, 1985.

[4] G. 't Hooft, "Strings from gravity," Physica Scripta, vol. 1987, no. 15 , article 143, 1987.

[5] G. 't Hooft, "The black hole interpretation of string theory," Nuclear Physics B, vol. 335, no. 1, pp. 138-154, 1990.

[6] G. 't Hooft, "The black hole horizon as a quantum surface," Physica Scripta, vol. 1991, no. 36, article 247, 1991.

[7] G. 't Hooft, "Scattering matrix for a quantized black hole," in Black Hole Physics, V. De Sabbata and Z. Zhang, Eds., pp. 381402, Kluwer, Dordrecht, The Netherlands, 1992.

[8] J. A. Harvey and A. Strominger, "Quantum aspects of black holes," in Proceedings of the 1992 TASI Summer School in Boulder, Colorado, University of Chicago report EFI-92-41, 1992.

[9] S. B. Giddings, "Toy models for black hole evaporation," in String Quantum Gravity and Physics at the Planck Energy Scale,
N. Sanchez, Ed., World Scientific, Singapore, University of California at Santa Barbara report UCSBTH-92-36, 1992.

[10] D. N. Page, "Black hole information," in Proceedings of the 5th Canadian Conference on General Relativity and Relativistic Astrophysics, University of Waterloo, 13-15 May, 1993, R. B. Mann and R. G. McLenaghan, Eds., World Scientific, Singapore, University of Alberta report Thy-23-93, 1994.

[11] G. 't Hooft, "Black hole unitarity and antipodal entanglement," Foundations of Physics, vol. 46, no. 9, pp. 1185-1198, 2016.

[12] E. Witten, "Anti de Sitter space and holography," Advances in Theoretical and Mathematical Physics, vol. 2, no. 2, pp. 253-291, 1998.

[13] G. 't Hooft, “The holographic principle," https://arxiv.org/abs/ hep-th/0003004.

[14] R. Bousso, "The holographic principle," Reviews of Modern Physics, vol. 74, no. 3, pp. 825-874, 2002.

[15] T. Banks, "Holographic space-time and its phenomenological implications," International Journal of Modern Physics A, vol. 25, no. 26, pp. 4875-4887, 2010.

[16] A. Y. Yosifov, "Quantum bubble polarization in curved spacetime," Journal of High Energy Physics, Gravitation and Cosmology, vol. 2, no. 2, pp. 244-252, 2016.

[17] A. Y. Yosifov and L. G. Filipov, "Black hole horizon oscillations," Aerospace Research in Bulgaria, no. 28, 2016.

[18] L. Susskind, L. Thorlacius, and J. Uglum, "The stretched horizon and black hole complementarity," Physical Review D, vol. 48, no. 8, pp. 3743-3761, 1993.

[19] R. Bousso, "A covariant entropy conjecture," The Journal of High Energy Physics, vol. 1999, no. 7, article 004, 1999.

[20] E. Bianchi, "Black hole entropy from graviton entanglement," https://arxiv.org/abs/1211.0522v2.

[21] P. Hayden and J. Preskill, "Black holes as mirrors: quantum information in random subsystems," Journal of High Energy Physics, vol. 2007, no. 9, article 120, 2007.

[22] A. Y. Yosifov and L. G. Filipov, "Oscillations for equivalence preservation and information retrieval from young black holes," Electronic Journal of Theoretical Physics, vol. 13, no. 36, pp. 197212, 2016.

[23] D. N. Page, "Information in black hole radiation," Physical Review Letters, vol. 71, no. 23, pp. 3743-3746, 1993.

[24] S. B. Giddings, "Models for unitary black hole disintegration," Physical Review D, vol. 85, no. 4, Article ID 044038, 2012.

[25] S. B. Giddings, "Nonviolent information transfer from black holes: a field theory parametrization," Physical Review D, vol. 88, no. 2, Article ID 024018, 2013.

[26] S. B. Giddings, "Modulated Hawking radiation and a nonviolent channel for information release," Physics Letters B, vol. 738, pp. 92-96, 2014.

[27] J. D. Bekenstein and V. F. Mukhanov, "Spectroscopy of the quantum black hole," Physics Letters B, vol. 360, no. 1-2, pp. 7-12, 1995.

[28] L. H. Ford and N. F. Svaiter, "Cosmological and black hole horizon fluctuations," Physical Review D, vol. 56, no. 4, pp. 2226-2235, 1997.

[29] L. H. Ford and N. F. Svaiter, "Gravitons and light cone fluctuations. II. Correlation functions," Physical Review D, vol. 54, no. 4, pp. 2640-2646, 1996.

[30] R. D. Sorkin, "How wrinkled is the surface of a black hole?" https://arxiv.org/abs/gr-qc/9701056vl. 
[31] R. D. Sorkin, “Two topics concerning black holes: extremality of the energy, fractality of the horizon," https://arxiv.org/abs/grqc/9508002v1.

[32] S. W. Hawking, "Black hole explosions?" Nature, vol. 248, no. 5443, pp. 30-31, 1974.

[33] S. D. Mathur and D. Turton, "The flaw in the firewall argument," Nuclear Physics B, vol. 884, no. 1, pp. 566-611, 2014.

[34] A. Saini and D. Stojkovic, "Radiation from a collapsing object is manifestly unitary," Physical Review Letters, vol. 114, no. 11, Article ID 111301, 2015.

[35] J. Hutchinson and D. Stojkovic, "Icezones instead of firewalls: extended entanglement beyond the event horizon and unitary evaporation of a black hole," Classical and Quantum Gravity, vol. 33, no. 13, Article ID 135006, 2016. 

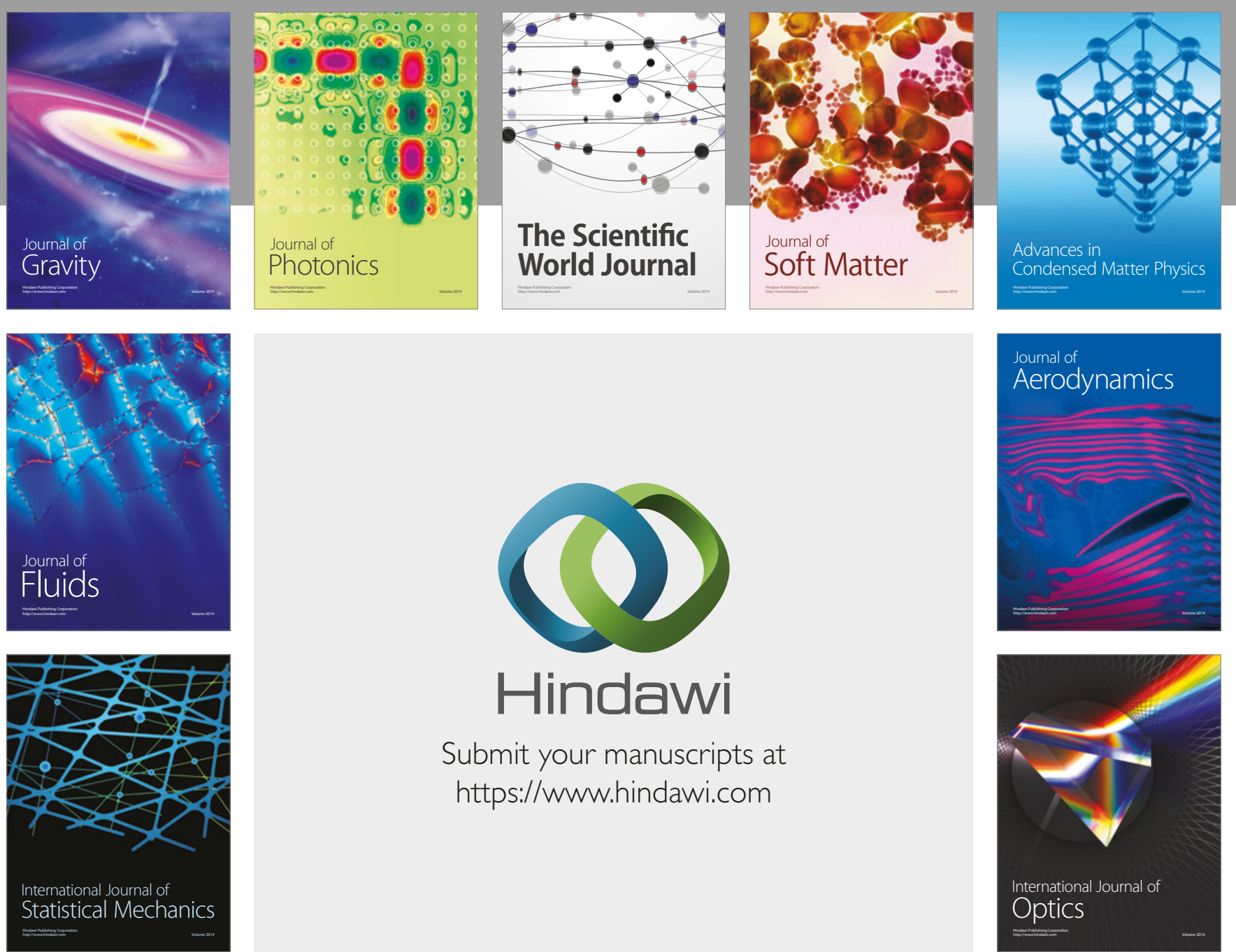

Submit your manuscripts at

https://www.hindawi.com
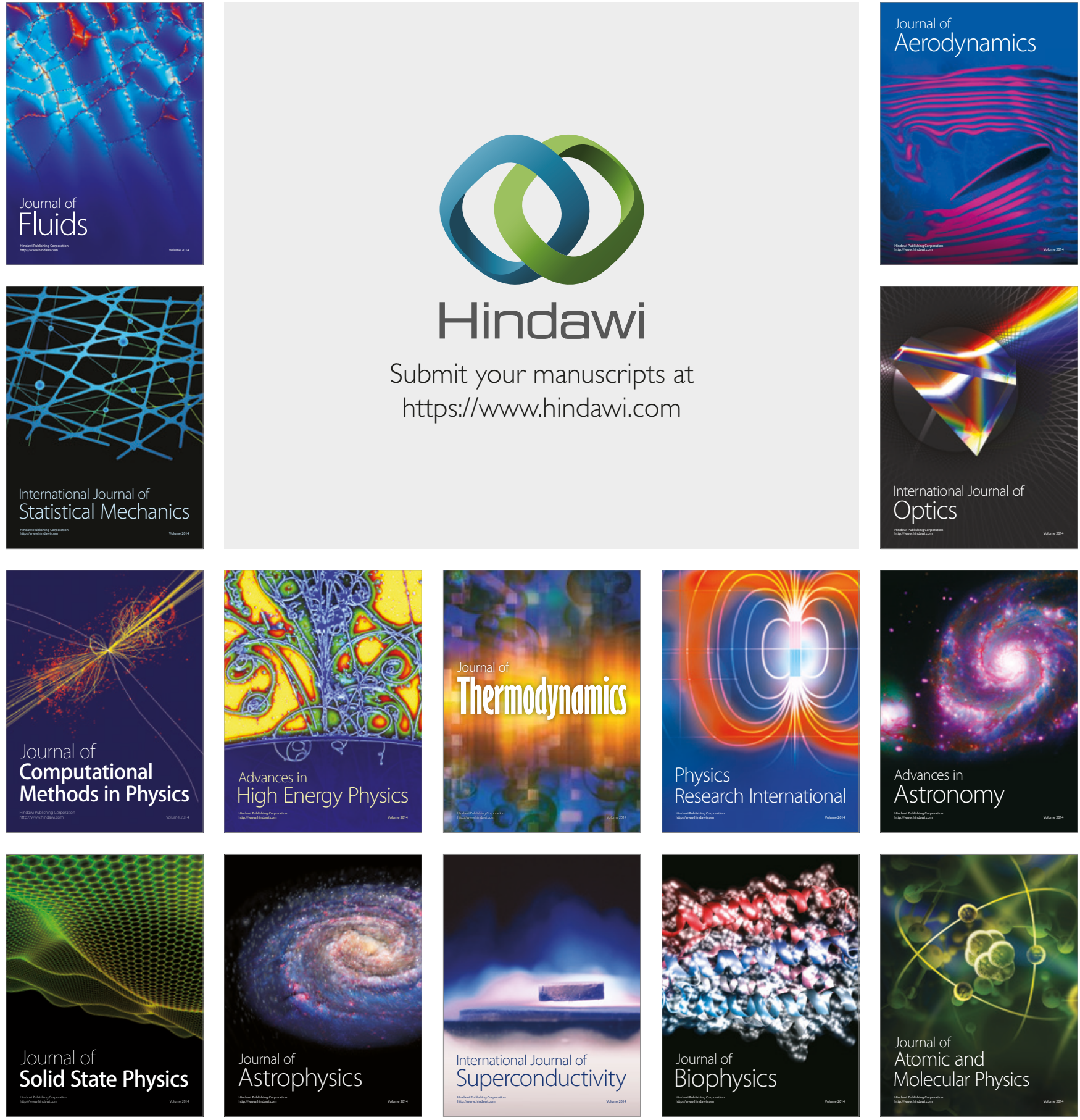